\title{
Correction to: Apolipoprotein E regulates lipid metabolism and a-synuclein pathology in human iPSC-derived cerebral organoids
}

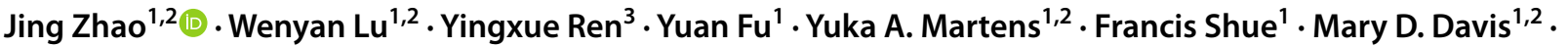 \\ Xue Wang ${ }^{3} \cdot$ Kai Chen ${ }^{1}$. Fuyao $\mathrm{Li}^{1}$. Chia-Chen Liu ${ }^{1}$ - Neill R. Graff-Radford ${ }^{4}$. Zbigniew K. Wszolek ${ }^{4}$. \\ Steven G. Younkin ${ }^{1}$. David A. Brafman ${ }^{5}$. Nilüfer Ertekin-Taner ${ }^{1,4} \cdot$ Yan W. Asmann ${ }^{3}$. Dennis W. Dickson ${ }^{1}$. Ziying Xu ${ }^{6}$. \\ Meixia Pan $^{6} \cdot$ Xianlin $\mathrm{Han}^{6,7} \cdot$ Takahisa Kanekiyo $^{1,2} \cdot$ Guojun Bu ${ }^{1,2}$ (D)
}

Published online: 1 November 2021

(c) The Author(s) 2021

\section{Correction to: Acta Neuropathologica (2021) 142:807-825 https://doi.org/10.1007/s00401-021-02361-9}

The original publication of this article contained an error in a Western blot image of Fig. 4b. The positive control lane of LIMP1 was mistakenly cropped when preparing the figure.
The correct image with positive control is now placed in the updated Fig. 4b. This error did not affect the results nor conclusion.

The corrected Fig. 4 is given below.

The original article can be found online at https://doi.org/10.1007/ s00401-021-02361-9.

\section{Guojun Bu}

bu.guojun@mayo.edu

1 Department of Neuroscience, Mayo Clinic, Jacksonville, FL 32224, USA

2 Center for Regenerative Medicine, Neuroregeneration Laboratory, Mayo Clinic, Jacksonville, FL 32224, USA

3 Department of Health Sciences Research, Mayo Clinic, Jacksonville, FL 32224, USA

4 Department of Neurology, Mayo Clinic, Jacksonville, FL 32224, USA

5 School of Biological \& Health Systems Engineering, Arizona State University, Tempe, AZ 85287, USA

6 Barshop Institute for Longevity and Aging Studies, University of Texas Health Science Center At San Antonio, San Antonio, TX 78229, USA

7 Department of Medicine, University of Texas Health Science Center At San Antonio, San Antonio, TX 78229, USA 
a
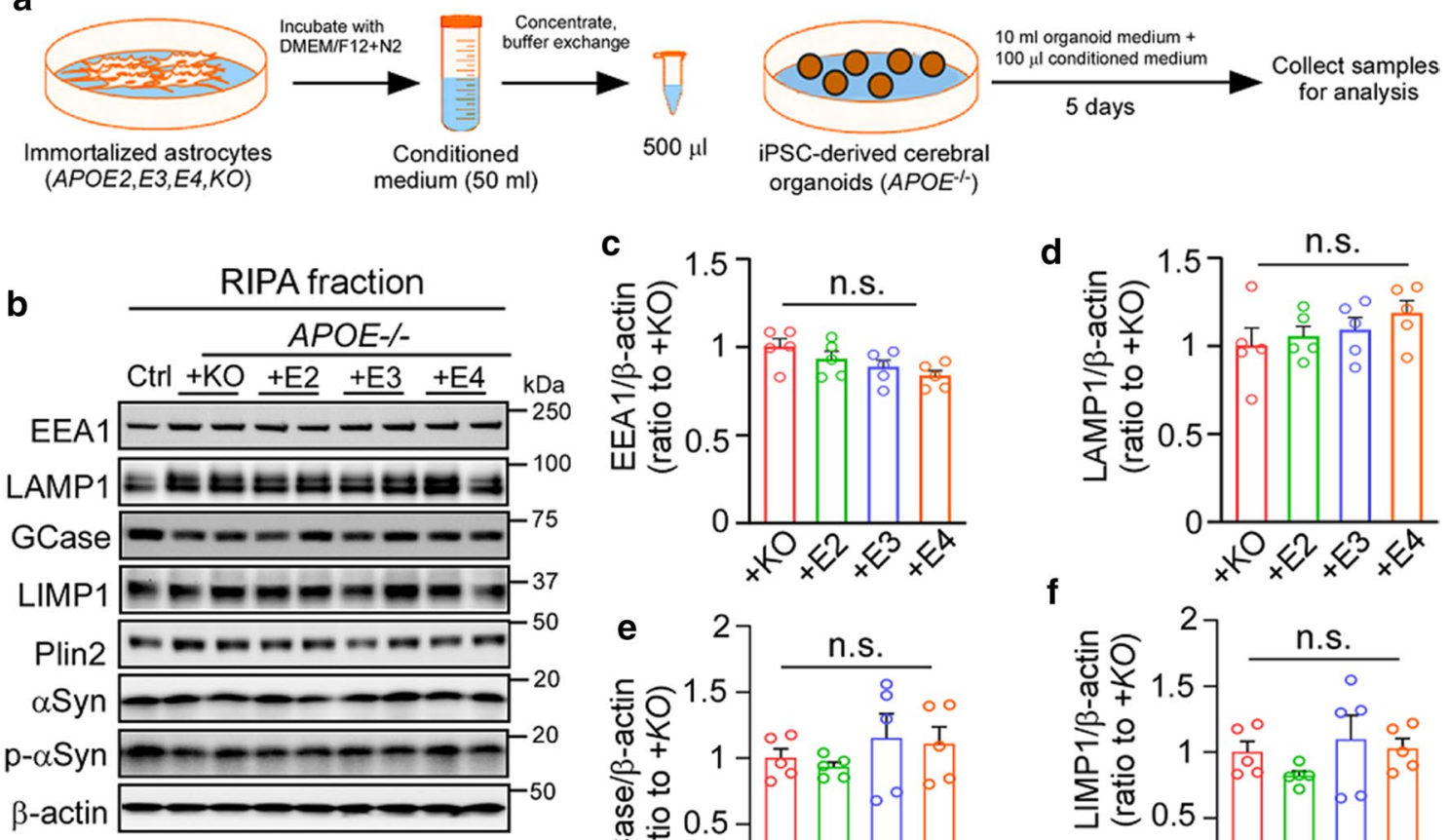

d
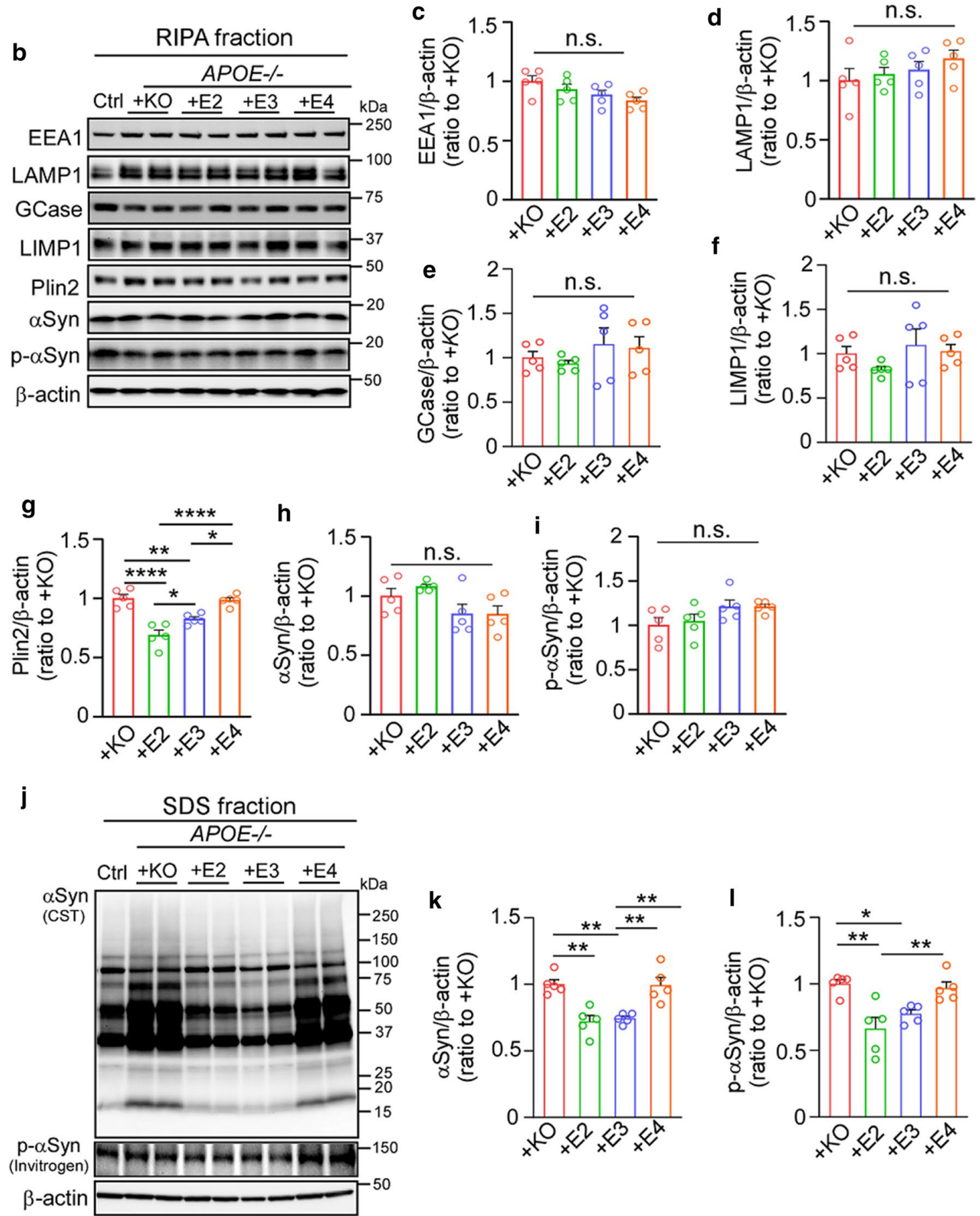
४Fig. 4 ApoE2 and apoE3, but not apoE4, partially rescue $\alpha$-synuclein accumulation in apoE-deficient cerebral organoids. The $A P O E^{-/-}$ iPSC-derived cerebral organoids at Day 90 were treated with conditioned media of immortalized astrocytes from APOE2-target replacement (TR), APOE3-TR, or APOE4-TR mice for 5 days. Conditioned media from Apoe-KO astrocytes were used as a control. a, A schematic workflow of the rescue experiments. b-i, Amounts of EEA1 (c), LAMP1 (d), GCase (e), LIMP1 (f), Plin2 (g), $\alpha$ Syn (h) and $p-\alpha$ Syn (i) in the RIPA fraction of the cerebral organoids after treatments were quantified by Western blotting. $\mathbf{j}-\mathbf{l}$, Amounts of total $\alpha \operatorname{Syn}(\mathbf{k})$ and $\mathrm{p}-\alpha \operatorname{Syn}(\mathbf{l})$ in the SDS fraction of cerebral organoids after treatments were quantified by Western blotting. All data were normalized to $\beta$-actin levels. 3 cerebral organoids were pooled and analyzed as one sample. All data are expressed as mean \pm SEM $(n=5$ samples/each). Experiments were repeated in three independent differentiation batches. One-way ANOVA was performed to determine statistical significance. ${ }^{*} p<0.01, * * * * p<0.0001$, n.s., not significant

Publisher's Note Springer Nature remains neutral with regard to jurisdictional claims in published maps and institutional affiliations. 